\title{
Laboreal
}

Volume $1 \mathbf{N}^{\circ} 1$ | 2005

Varia

\section{Uso de los recursos, adaptación de los conocimientos y gestión de la autonomia en la decisión terapéutica}

Uso dos recursos, adaptação dos saberes e gestão da autonomia na decisão terapêutica

Usage des ressources, adaptation des savoirs et gestion de l'autonomie dans la décision thérapeutique

Resources use, knowledge adaptation and autonomy management in therapeutic decision

\section{Vanina Mollo}

\section{CpenEdition}

\section{Journals}

Edición electrónica

URL: http://journals.openedition.org/laboreal/14080

DOI: 10.4000/laboreal. 14080

ISSN: 1646-5237

Editor

Universidade do Porto

Referencia electrónica

Vanina Mollo, « Uso de los recursos, adaptación de los conocimientos y gestión de la autonomia en la decisión terapéutica », Laboreal [En línea], Volume 1 Nº1 | 2005, Publicado el 01 diciembre 2005, consultado el 23 septiembre 2020. URL : http://journals.openedition.org/laboreal/14080 ; DOI : https://doi.org/10.4000/laboreal.14080

Este documento fue generado automáticamente el 23 septiembre 2020

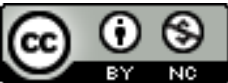

Laboreal está licenciado com uma Licença Creative Commons - Atribuição-NãoComercial 4.0 Internacional. 


\section{Uso de los recursos, adaptación de los conocimientos y gestión de la autonomia en la decisión terapéutica}

Uso dos recursos, adaptação dos saberes e gestão da autonomia na decisão terapêutica

Usage des ressources, adaptation des savoirs et gestion de l'autonomie dans la décision thérapeutique

Resources use, knowledge adaptation and autonomy management in therapeutic decision

\section{Vanina Mollo}

\section{REFERENCIA}

Mollo, V. (2005). Usage des ressources, adaptation des savoirs et gestion de l'autonomie dans la décision thérapeutique. Thèse de Doctorat en Ergonomie, Laboratoire d'ergonomie du CNAM, Paris.

\section{NOTA DEL EDITOR}

Manuscrito recibido en : agosto/2005

Aceptado tras peritage en : octubre/2005

1 En muchas situaciones de trabajo, los operadores se ven sometidos a reglas más o menos restrictivas a las cuales se deben referir para tomar decisiones, y que tienen generalmente por objetivo asegurar la calidad y la fiabilidad de la producción, tanto si 
esta se reporta a un producto o a un servicio. Esta voluntad de prescribir la actividad de los operadores suscita dos cuestiones, una relativa al estatuto de las desviaciones a las reglas, la otra a la autonomía de los operadores.

Pueden distinguirse dos modelos de utilización de las reglas (Dekker, 2003). El primero considera las reglas como el medio más seguro de asegurar la calidad y la fiabilidad de la producción. El comportamiento esperado de los operadores es entonces el de conformarse a las reglas. En el caso contrario, las acciones de mejora consistirán en asegurarse que los operadores conocen bien las reglas y que las siguen. El segundo modelo, por otro lado, considera las reglas como recursos incompletos, lo que significa que desde su concepción, ellas no consideran todas las situaciones encontradas en la práctica cuotidiana. El comportamiento esperado de los operadores consiste no en conformarse estrictamente a las reglas existentes, pero en determinar cuando y como adaptarse. Si ellos fallan aplicando una regla que no está adaptada a la situación o intentando una adaptación que falla, las acciones de mejora visarán controlar y comprender los diferentes tipos de desviaciones, a fin de definir las situaciones críticas y asistir los operadores en su juicio de adaptabilidad, para mejorar su capacidad de juzgar cuando y como adaptar.

3 Según el modelo considerado, el espacio atribuido a la autonomía de los operadores difiere. En el primer modelo, la autonomía constituye una fuente de infiabilidad pudiendo poner en peligro el buen funcionamiento del sistema, mientras que en el segundo, ella es un recurso indispensable a la decisión.

4 Es en esta segunda perspectiva que se inscribe este trabajo. La autonomía se define entonces como "la capacidad en discernir, en función de las situaciones o de los contextos, el carácter obligatorio, facultativo, inútil, hasta a veces perjudicial de las reglas, y en hacer variar la sumisión en función de las contingencias" (Everaere, 1999).

5 Estas cuestiones relativas al estatuto de las desviaciones a las reglas y al espacio atribuido a la autonomía de los operadores han sido tratadas en el contexto de la normalización de las prácticas médicas, y más particularmente en oncología, donde se pasó progresivamente de una medicina fundada sobre el peritaje clínico específico de los especialistas a la medicina fundada sobre las evidencias (Evidence-based Medicine : EBM), definida como "el uso consciente, explícita y juiciosa de la mejor prueba disponible relativamente al cuidado de los pacientes" (Sackett, Rosenberg, Gray, Haynes \& Richardson, 1996). Los médicos disponen de referenciales terapéuticos sobre los cuales se deben apoyar para tomar las decisiones de tratamientos. Estos referenciales compilan, bajo la forma de reglas, los resultados publicados con base en ensayos terapéuticos (Evidence-based working group, 1992); así, tomar una decisión implica categorizar los casos a partir de las reglas existentes y aplicar la acción correspondiente.

6 El objetivo de una tal normalización es de mejorar la calidad de los cuidados administrados a los pacientes y de homogeneizar las prácticas. Pero a pesar de su utilidad manifiesta, el EBM no considera la variabilidad de los pacientes, tanto sobre el plano médico (contra-indicaciones medicamentosas) como en el plano de las necesidades y preferencias, los pacientes pueden emitir pedidos particulares que limitan la aplicabilidad de los tratamientos (por ejemplo : el rechazo de un tratamiento) (Knottnerus \& Dinant, 1997; Howitt \& Armstrong, 1999 ; Bensing, 2000 ; Freeman \& Sweeney, 2001 ; Haynes, 2002). Este carácter incompleto de las reglas ha conducido al desarrollo de Comités de Concertación Pluridisciplinarios (CCP). Se trata de reuniones 
de especialistas cuyo objetivo es proveer un parecer sobre casos que comportan elementos perturbadores, es decir factores que impiden la aplicación stricto sensu del referencial.

El presente estudio, realizado en un centro oncológico, aborda estas situaciones de desviación a las reglas, y visa comprender el proceso de adaptación. Se trata de poner en evidencia los recursos utilizados por los médicos para tratar los casos fuera del referencial (experiencia pasada, CCP, colegas), y de analizar el proceso de construcción de la autonomía, es decir, de colocar por delante el estatuto de las reglas en la decisión y de analizar el impacto del colectivo sobre las elecciones individuales. De hecho, si este último es parte integrante de la actividad de los médicos (atestiguando la existencia de los CCP), él no permite hacer sobresalir los efectos de la actividad colectiva sobre el individuo. Es por esta razón que una parte importante de este trabajo consistió en simular la actividad del CCP, por la confrontación de los médicos con las decisiones de sus pares, para analizar sus efectos sobre el aprendizaje individual.

Para responder a estos objetivos, se puso en operación una metodología de extracción de los conocimientos de adaptación. Ella se desarrolló en dos fases. En la primera, 19 médicos (6 radioterapeutas, 6 cirujanos, 6 oncólogos y 1 ginecólogo), vistos individualmente, solucionaron en voz alta 14 problemas realistas implicando uno o varios elementos perturbadores. Estos últimos corresponden a tres tipos de incertidumbres :

1. - La incertidumbre sobre la caracterización del problema: la dificultad asienta en la inclusión del problema en una categoría del referencial. Se puede tratar de valores que no se tienen en cuenta por el referencial (por ejemplo, no existe referencial relativo al cáncer de mama del hombre), de valores situados en el limite de los valores de una clase (edad, tamaño del tumor), o de datos definitivamente en falta (localización desconocida del tumor en la mama).

2. - La incertidumbre sobre las características médicas del paciente, que comprende esencialmente los antecedentes médicos (embolia pulmonar) y las patologías asociadas del paciente (hipertensión), pero también ciertas características físicas que contra-indican ciertos tratamientos (embarazo).

3. - La incertidumbre relativa a la elección del paciente: se trata de pedidos específicos formulados por el paciente (rechazo de un tratamiento, deseo de quedarse embarazada).

Los médicos tienen una experiencia mínima de 10 años, y pertenecen a la misma red de cuidados regional. Resolver los problemas significaba analizar las características específicas del problema colocado, considerar las soluciones terapéuticas potenciales y jerarquizarlas. Estos problemas han sido construidos en colaboración con un especialista del centro y se referían no al diagnóstico, pero sí a la elección de una terapéutica.

10 La segunda fase consistió en efectuar las allo-confrontaciones individuales : 13 de los 19 médicos fueron confrontados individualmente con las soluciones de sus pares a fin de que ellos las comentasen (Mollo \& Falzon, 2003; 2004). Estas soluciones fueron completadas por los extractos de conversaciones, para que los médicos tuviesen acceso a las justificaciones de las elecciones de sus colegas. Este proceso de evaluación contrastante permite, por un lado, una mayor clarificación de los conocimientos recogidos en la primera etapa, y por otro lado, acceder a los efectos subyacentes del proceso de confrontación puesto en práctica en el CCP, simultáneamente sobre el desarrollo de los conocimientos individuales y colectivos. En esta segunda fase, que 
exige más tiempo que la primera, y no pudiendo el tiempo concedido por médico exceder $1 \mathrm{~h}$, fueron retenidos 6 problemas con base en la diversidad observada al nivel de las soluciones propuestas (número de soluciones diferentes propuestas por problema y reparto de los efectivos asociados a cada solución) y en el grado de divergencia entre las diferentes soluciones (para ciertos problemas, las soluciones propuestas variaban de la prescripción de un tratamiento a la supresión de ese tratamiento). Los extractos de conversaciones fueron seleccionados con base en la variabilidad de los criterios de decisión utilizados por los médicos.

11 En el final de cada problema tratado, los médicos debían validar o modificar las soluciones que ellos habían indicado en la primera fase a fin de analizar los cambios eventuales de soluciones.

El análisis de datos orales así recogidos hizo destacar dos resultados principales.

El primero atesta que los instrumentos que visan normalizar las prácticas médicas y la autonomía de los médicos constituyen menos constreñimientos que los recursos que los médicos utilizan a su manera, en función de sus necesidades (Castel \& Merle, 2002). El referencial terapéutico formaliza, bajo la forma de reglas, los conocimientos provenientes de la literatura científica, facilitando así el proceso de pesquisa de solución. Mismo en una situación no habitual, él sirve igualmente de base al raciocinio de los médicos. Esta afirmación asienta sobre dos constataciones. Por un lado, los elementos perturbadores han sido identificados por todos los médicos, lo que muestra que estos reconocen la desviación a la regla, y por lo tanto que esta última constituye su referencia. Por otro lado, una vez reparada la desviación, los médicos reflejan sobre la base del referencial que sería aplicado en la ausencia de los elementos perturbadores. Así, cuando una situación dada pone en causa la aplicabilidad del referencial, este no es abandonado pero sí adaptado. Es por eso que hablamos de una adaptación reflexionada (Sauvagnac, 2000). Sin embargo, el universo del referencial estando exclusivamente centrado sobre la patología, se hace insuficiente. Los médicos deben necesariamente integrar criterios provenientes de otros universos, designadamente el universo de los pacientes (estado clínico y psicológico, creencias...), el universo técnico-organizacional (recursos materiales, organizacionales y temporales), el universo colectivo (colegas, CCP) y el universo del propio médico, que comprende al mismo tiempo los conocimientos advenidos de la pesquisa clínica y la práctica efectiva. Relativamente al CCP, este ofrece a los médicos un medio de reducir la incertidumbre a la cual ellos pueden ser confrontados. Dicho esto, él no es exclusivo : los médicos pueden admitir un parecer junto de sus colegas más que en el CCP, y este último puede ser utilizado para otros fines que el de la ayuda a la decisión (apoyo médico-legal, aprendizaje colectiva, etc.).

14 Así, a pesar de los instrumentos que se les imponen, los médicos disponen de una autonomía consecuente que les permite gestionar el "potencial de sorpresa" característico de todo el dominio de actividad (Woods \& Shattuck, 2000), y, al hacerlo, completar las reglas existentes especificando sus valores o creando nuevas reglas adaptadas a los casos particulares.

El segundo resultado se refiere al impacto del colectivo (CCP) sobre la construcción y desarrollo de la autonomía. Esta asume un papel importante en la determinación de soluciones inaceptables por un lado, ciertas soluciones han sido consideradas inadecuadas por la mayoría de los médicos que las comentaron y, por otro lado, en la determinación de las soluciones aceptables : al contrario del punto precedente, otras 
soluciones son consideradas aplicables por la mayoría de los médicos que las evaluaron, a pesar de no hacer parte de sus elecciones iniciales.

En fin, la confrontación con las decisiones de otro representa un papel importante en el cambio de soluciones ya que $48 \%$ de los casos, los médicos modificaron su elección inicial.

El CCP permite por lo tanto la construcción de un referencial local para las situaciones no habituales, y al hacerlo, participa en el desarrollo del espacio de autonomía de los médicos. Su objetivo consiste más en definir el espacio de soluciones posibles e imposibles que se aproximan de una solución única aceptables (aunque esto pueda talvez ser necesario). Así, él provee un cuadro de referencia a los médicos que les permite efectuar las elecciones según sus propias reglas de especialista, asegurando la fiabilidad de las decisiones tomadas (Mollo, 2004). El CCP es igualmente un instrumento de difusión de las reglas locales y participa en el aprendizaje de los médicos, los cuales han sido llevados a integrar las reglas construidas, en el decurso de la confrontación.

El conjunto de estos elementos conduce al ergónomo a interrogarse sobre los medios de favorecer el papel del especialista clínico específico de los médicos en la evolución de las reglas y, más particularmente, sobre los instrumentos susceptibles de apoyar la construcción y el desarrollo de conocimientos. De hecho hasta aquí, los efectos inducidos sobre las personas y sobre el propio colectivo se han hecho implícitos, estando el CCP más centrado sobre la producción de decisiones adaptadas a los casos particulares que sobre la construcción y desarrollo de los conocimientos. La alloconfrontación individual podría constituir un instrumento de soporte a esta actividad reflexiva. El interés de este método es que él desencadena un cambio de representación que resulta del hecho del operador estar colocado lejos de su propia actividad; el confronto con los conocimientos del otro lo conduce así a una mejor justificación de sus propias reglas de decisiones. Sin embargo, este método integrado en la perspectiva de evolución de los conocimientos presenta dos límites esenciales. Por un lado, la evolución del referencial no puede ser encarada sino a partir de una reflexión colectiva (y no individual) sobre los diferentes problemas encontrados en la práctica. Por otro lado, aunque la adaptación local de una regla pueda ser considerada como la premisa de una nueva regla, es la repetición de formas de adaptación similares que permite evolucionar hacia el desarrollo de nuevas reglas. Es por lo tanto necesario disponer de un número suficiente de casos similares. Una de las vías posibles es el análisis de los casos tratados en el CCP, que permitiría la comparación de estos casos en función del tipo de soluciones propuestas para un mismo problema. Una solución es entonces juntar las alloconfrontaciones individuales y el CCP: los médicos tratarían primeramente solos un problema, después expondrían su raciocinio a sus colegas, debiendo discutir la lógica de raciocinio y los diferentes tratamientos propuestos. La allo-confrontación adquiere así el estatuto de metodología para la actividad reflexiva colectiva.

19 Es preciso, finalmente, recordar que además de los recursos internos a la organización, la decisión terapéutica es un proceso de cooperación entre el médico y el paciente cuya gestión es dejada a la total autonomía del médico. El análisis de los datos muestra que los conocimientos que los médicos disponen sobre los pacientes encontrados a lo largo de su práctica están en el origen de ciertas soluciones de adaptación, sin que estos hayan formulado un pedido particular. Ella muestra igualmente que el estilo adoptado por los médicos en la relación depende fuertemente del grado de accesibilidad de los 
pedidos. Estos resultados proveen bases de reflexión relativas a la participación de los pacientes en el proceso de decisión, que constituye una problemática cada vez más desarrollada en el dominio de la salud. Ellos conducen, designadamente, a pensar que los pedidos de los pacientes, en ciertas situaciones (y bajo ciertas condiciones), podrían ser introducidos en los referenciales terapéuticos, a fin de ser considerados en la decisión (Flatley, Brennan \& Strombom, 1998 ; Protheroe, Fahey, Montgomery \& Peters, 2000).

\section{BIBLIOGRAFÍA}

Bensing, J.P.D. (2000). Bridging the gap. The separate worlds of evidence-based medicine and patient-centered medicine. Patient Education and Counseling, 39, (1), 17-25.

Castel, P., \& Merle, I., (2002). Quand les normes de pratiques deviennent une ressource pour les médecins. Sociologie du Travail, 44, (3), 337-355.

Dekker, S. (2003). Failure to adapt or adaptations that fail : contrasting models on procedures and safety. Applied Ergonomics, 34, (3), 233-238.

Everaere, C. (1999). Autonomie et collectifs de travail. Lyon : ANACT. Evidence-based medicine working group (1992). Evidence-based medicine. A new approach to teaching practice for medicine. JAMA, 268, (17),2420-2425.

Flatley Brennan, P., \& Strombom, I. (1998). Improving health care by understanding patient preferences. The role of computer technology. Journal of the American Medical Informatics Association, 5, (3), 257-262.

Freeman, A.C., \& Sweeney, K. (2001). Why general practitioners do not implement evidence : qualitative study. British Medical Journal, 323 (7303), 1-5.

Haynes, R.B. (2002). What kind of evidence is it that evidence-based medicine advocates want health care providers and consumers to pay attention to ? BMC Health Services Research, 2, (3), $7 \mathrm{p}$.

Howitt, A., \& Armstrong, D. (1999). Implementing evidence-based medicine in general practice : audit and qualitative study of antithrombotic treatment for atrial fibrillation. British Medical Journal, 318, (7194), 1324-1327.

Knottnerus, A., \& Dinant, G.J. (1997). Medicine based evidence, a prerequisite for evidence based medicine. British Medical Journal, 315 (7116), 1109-1110.

Mollo, V., \& Falzon, P. (2003). Formalizing confrontation methods : a categorization attempt. Proceedings of the XVth Thriennial Congress of International Ergonomics Association, "Ergonomics in the Digital Age", August 24-29, 2003 Seoul, Korea, 4 p.

Mollo, V. (2004). Normalisation et adaptation des règles en cancérologie. In P. Rey, E. Ollagnier, V. Gonik \& D. Ramaciotti (Eds.) Communication au XXXIXème Congrès de la SELF, "Ergonomie et normalisation" : 15-17 septembre 2004, Genève (pp. 221-229). Toulouse : Octarès. 
Mollo, V., \& Falzon, P. (2004). Autoand allo-confrontation as tools for reflective activities. Applied Ergonomics, 35, (6), 531-540.

Protheroe, J., Fahey, T., Montgomery, A.A., \& Peters, T.J. (2000). The impact of patients' preferences on the treatment of atrial fibrillation : observational study of patient based decision analysis. British Medical Journal, 320, 1380-1384.

Sackett, D.L., Rosenberg, W.M.C., Gray, J.A.M., Haynes, R.B., \& Richardson, W.S. (1996). Evidencebased medicine, what it is, and what it isn't. British Medical Journal, 312, (7023), 71-72.

Sauvagnac, C. (2000). La construction de connaissances par l'utilisation et la conception de procédures. Contribution au cadre théorique des activités métafonctionnelles. Thèse de doctorat en Ergonomie, CNAM, Paris.

Woods, D.D. \& Shattuck, L.G. (2000). Distant supervision local action given the potential for surprise. Cognition, Technology and Work, 2, (4), 242-245.

\section{AUTOR}

VANINA MOLLO

Laboratoire d'Ergonomie du CNAM, 41 rue Gay Lussac, 75005 Paris, France

vanina.mollo@cnam.fr 